10 - ORIGINAL ARTICLE

TECHNICAL SKILL

\title{
Simulation-based ultrasound-guided central venous cannulation training program ${ }^{1}$
}

\author{
Rafael Denadai, Andreia Padilha Toledo" ${ }^{\mathrm{II}}$, Danielle Milani Bernades ${ }^{\mathrm{III}}$, Felipe Daldegan Diniz ${ }^{\mathrm{III}}$, Fernanda Brandão Eid ${ }^{\mathrm{III}}$,

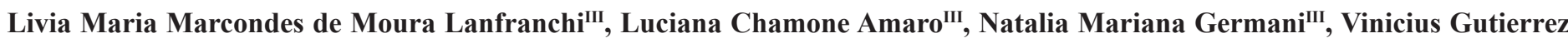 \\ Parise $^{\text {III }}$, Claudio Nascimento Pacheco Filho ${ }^{\text {IV }}$, Rogério Saad-Hossne ${ }^{\mathrm{V}}$ \\ ${ }^{\mathrm{I} M D}$, Resident, Department of Surgery, Hospital Municipal Dr. Mario Gatti (HMMG), Campinas-SP, Brazil. Conception, design, intellectual and \\ scientific content of the study; technical procedures; manuscript writing, critical revision. \\ ${ }^{I}$ MD, Resident, Department of Internal Medicine, Faculty of Medicine, Sao Paulo University (FMUSP), Sao Paulo-SP, Brazil. Technical procedures, \\ acquisition of data, manuscript writing. \\ IIIMD, Resident, Department of Surgery, HMMG, Campinas-SP, Brazil. Technical procedures. \\ ${ }^{\mathrm{IV}} \mathrm{MD}$, Surgeon-in-Chief, Department of Surgery, HMMG, Campinas-SP, Brazil. Technical procedures. \\ ${ }^{v} \mathrm{PhD}$, Associate Professor, Division of Coloproctology, Department of Surgery, Botucatu Medical School (FMB), State University of Sao Paulo \\ (UNESP), Botucatu-SP, Brazil. Manuscript writing, critical revision, supervised all phases of the study.
}

\section{ABSTRACT}

PURPOSE: To propose a simulation-based ultrasound-guided central venous cannulation skills' training program, during residency.

METHODS: This study describes the strategies for learning the ultrasound-guided central venous cannulation on low-fidelity bench models. The preparation of bench models, educational goals, processes of skill acquisition, feedback and evaluation methods were also outlined. The training program was based on key references to the subject.

RESULTS: It was formulated a simulation-based ultrasound-guided central venous cannulation teaching program on low-fidelity bench models.

CONCLUSION: A simulation-based inexpensive, low-stress, no-risk learning program on low-fidelity bench models was proposed to facilitate acquisition of ultrasound-guided central venous cannulation skills by residents-in-training before exposure to the living patient. Key words: Education. Models, Educational. Learning. Residency. Surgery. Teaching Materials. Ultrasonography. Ultrasonography, Interventional. Vascular Access Devices. 


\section{Introduction}

About 5 million central venous cannulations (CVC) aimed at obtaining central venous access are placed annually in the United States in a variety of settings, including surgical intensive care $^{1}$. CVC has historically been performed "blindly" using only palpable anatomic landmarks such as bony prominences, muscle surfaces, and arterial pulsations ${ }^{2}$. This "blind" approach assumes anatomic homogeneity and depends on correct discernment of the relationship among multiple anatomic landmarks ${ }^{2}$. However, approximately $10 \%$ of patients have anatomical variations and this factor may account for complications (accidental arterial puncture, multiple attempts to achieve success, malposition of the catheter tip, hematoma, pneumothorax, and hemothorax) using the anatomic landmark technique alone ${ }^{1,2}$. Complication rates associated with the landmark-based approach have been reported to be as high as $18.8 \%$ and they can be categorized as patient dependent (anatomic variation, body habitus, and coagulopathy) or operator dependent (time allotted to perform the procedure, fatigue, operator's level of experience, and lack of ultrasound [US] guidance) $)^{2}$. Operatordependent factors (mainly, the operator's level of experience and the lack of US guidance) are of special interest since they can be targets for an education intervention with the ultimate aim of improving patient care $^{1,2}$.

Since the first reports on the use of two-dimensional (2D) external US for CVC, the rationale for the use of US to guide $\mathrm{CVC}$ is robust ${ }^{3,4}$. An extensive body of evidence-based literature ${ }^{3,4}$ has showed that the US-based approach is associated with fewer complications, fewer attempts before successful cannulation, fewer failed procedures, and shorter procedure times when compared to the historical anatomic landmark approach. Following these publications ${ }^{3,4}$, various professional medical organizations and government agencies such as the American College of Surgeons (ACS), College's Committee on Perioperative Care, American Society of Anesthesiologists (ASA), Agency for Healthcare Research and Quality (AHRQ), National Institute of Clinical Excellence (NICE), American Society of Echocardiography, and Society of Cardiovascular Anesthesiologists have issued statements advocating that 2-D US guidance should be the preferred method for CVC in both adults and children in elective situations, and should be considered in most clinical circumstances where CVC insertion is necessary in an emergency situation ${ }^{5}$. Unfortunately, the incorporation of these recommendations into clinical practice has faced a lot of resistance ${ }^{3}$.

Among the major obstacles (e.g., lack of US equipment, the purchase costs of the US machines, time required, and lack of familiarity with the technology due to lack of experience or training $)^{3}$ to the widespread implementation of US guidance in CVC procedures, lack of formal training is particularly relevant because it has been demonstrated a negative correlation between the frequency of complications and the operator experience ${ }^{1,6}$. Moreover, as in 2011 the Healthcare Infection Control Practices Advisory Committee of Centers for Diseases Control and Prevention (CDC, USA Government) indicated that US-guided central venous access placement could be performed only by those who are properly trained ${ }^{7}$, all efforts should be focused on training those physicians (e.g., residents, emergency physicians, among others) who may potentially be faced with clinical situations that require $\mathrm{CVC}^{8}$.

Residents commonly perform $\mathrm{CVC}^{9}$ and competency in this technical skill is a stated objective for a number of postgraduate medical training programs such as the Royal College of Physicians and Surgeons of Canada and the Joint Royal Colleges of Physicians Training Board of United Kingdom ${ }^{9}$ However, despite these efforts for improving the training of this skill, many residents feel uncomfortable when performing invasive procedures, including $\mathrm{CVC}^{9,10}$. Additionally, although residents have reported that the use of US was one of the most important factors to avoid bedside procedural complications ${ }^{9,10}$, the US-guided technique requires knowledge of ultrasonography and hand-eye coordination, and most residents have little formal training in these fields ${ }^{10}$. Thus, given the apparent gap between regulatory expectations and actual performance, the development of alternatives for this training is of utmost importance ${ }^{4}$.

Traditional education in central venous access insertion usually involves learning at the bedside of living patients without opportunity for prior standardized deliberate step-by-step practice or skills' assessment ${ }^{11}$. Bearing in mind that this learning on living patients violates bioethical concerns ${ }^{12}$, simulation-based education may play a role in addressing these needs for teaching and learning ${ }^{13}$. The possibility of performing such invasive procedures on nonhuman simulators has the potential to provide the necessary skill to establish best-practice recommendations for US use and, ultimately, to improve patient safety ${ }^{11}$.

Several reports ${ }^{8,11}$ have demonstrated that the simulationbased US-guided CVC training improves the overall success of central venous catheter insertion and there is a general consensus that formal education and training are necessary ${ }^{5}$. However, recent studies $^{4,8,11}$ have showed that the ideal training methodology is unclear and there is lack of harmony for standards of US-guided CVC training, making the format of the teaching extremely variable. Moreover, despite the importance of this issue, the body 
of national (Brazilian) literature is small. Most national studies ${ }^{14,15}$ addresses only the technical aspects on the use of US technology, leaving the desired on simulation-based US-guided CVC teaching for residents-in-training.

As there is a need to establish recommendations on education, training, competence, and proficiency in US-guided central venous access placement $t^{4,8}$ and as new opportunities in simulation-based education need to be explored to positively impact quality and safety in surgical care ${ }^{12}$, the purpose of this study was to describe a simulation-based inexpensive, lowstress, no-risk learning program on low-fidelity bench models to facilitate acquisition of US-guided CVC skills to enhance the educational experiences of residents-in-training and ensure adequate preparation before living patient exposure. Additionally, this report is aimed at serving as a guide for both residents-intraining and instructors. Thus, this study is intended to include only selected references of importance, but it is not meant to be a comprehensive or rigorous literature review, as this has been accomplished elsewhere ${ }^{3,4}$.

\section{Simulation-based ultrasound-guided central venous cannulation skills training}

Simulation-based education recently emerged as an important learning tool in several areas of surgical expertise ${ }^{12,16}$ and it is particularly attractive for teaching invasive procedures such as CVC, which require eye-hand coordination and ambidextrous maneuvers ${ }^{4,11}$. Simulation allows residents-intraining to repeatedly practice a procedure prior to performing it on a living patient and also has potential benefits to improve provider performance, to reduce errors, and, ultimately, to enhance patient safety ${ }^{11}$. Although there is a lack of consensus for teaching and learning in US-guided $\mathrm{CVC}^{4}$, the use of simulation-based USguided CVC training should be considered the standard of care because recently some studies ${ }^{8,13}$ demonstrated the efficacy and usefulness of simulation-based training in CVC.

The proposed educational program is based on selfdirected training and feedback from instructors, distributed in several sessions of teaching and learning ${ }^{16,17}$. As previous report ${ }^{8}$ showed that didactic training combined with simulation-based training consisting of video instruction followed by supervised performance on nonhuman simulators is superior to traditional training, didactic training alone, or video training alone for improving US-guided CVC skills, each session consists of steps to be undertaken in subsequent ways: 1) verbal teaching supervised by instructor and based on on-line narrated expert demonstration videos (indications, contraindications, complications, and basic technique for CVC - demonstration of normal human vascular anatomy by using US visualization on a volunteer and also the aspect of the simulated vessel on bench models); 2) self-directed hands-on-training on bench models with immediate and posterior feedback from the instructor in the simulated operating setting; 3) self-directed hands-on-training on bench models with posterior feedback from the instructor focused on extra-laboratory training; 4) and trainees are required to make the central venous catheter placement in a real patient care setting under the instructors' supervision during the last months of their first year of residency ${ }^{1,8,10}$. Regardless of the feedback, it is important to encourage trainees to clear up their doubts during all training phases. Moreover, each training step can be repeated according to the individual needs.

\section{Educational goals}

Once the basic skills training can lead to improved performance of more complex tasks, it is important to teach all steps in an organized sequence ${ }^{17}$. The process breaks down the CVC procedure into all of its parts and then puts the different components together. To do this, it is important to include educational goals that are set before the beginning of the teaching and learning process $^{16,17}$. During the training steps, the instructor should explain the advantages and disadvantages of each technical aspect and the proper choice/use of materials. In this training program, it is included the US-guided CVC skills as follows: preparation of equipment, use of US technology (on/off button, freeze, gain, depth, and measure), handling US transducers (probe orientation), maintenance of aseptic technique (to achieve competence in creating and maintaining a wide sterile barrier, including the application of a sterile US probe sleeve), identification of vascular anatomy (to find the simulated vessel), placement of centre of US probe over centre of simulated vein, visualization of needle-tip approaching and penetrating the simulated vein, and confirmation of successful puncture by aspiration as well as visualization ${ }^{3,10}$. After mastering these simulated procedures, trainees can perform US-guided CVC on living patients.

\section{Training time}

There are no clear recommendations on the total number of hours that trainees must practice to acquire US-guided CVC skills. In fact, The American College of Emergency Physicians ${ }^{18}$ recommend that all US practitioners should undergo initial basic US training varying in length from 1 to 2 days depending on 
the number of applications being taught, followed by at least 25 documented and reviewed cases of primary applications including US-guided procedures such as CVC, pericardiocentesis, paracentesis, among others. However, it is unclear for how many hours of specific CVC training should be performed ${ }^{18}$.

Thus, as described by others ${ }^{6,8,10}$ we adopt the following training period: on-line narrated expert demonstration videos session (1 hour to 2 hours); self-directed hands-on-training in the laboratory of simulation session (approximately 2 hours); and self-directed extra-laboratory hands-on-training session (deliberate practice and self-controlled by the trainee). After mastering these simulated skills, trainees should perform complete procedures on living patients; the initial CVC (at least 5 to ten proctored procedures) $)^{6,19}$ should be supervised by the instructor to the fidelity level when compared to a live human being, there are high-fidelity simulators such as parts of postmortem animals (turkey and chicken breasts, pieces of pork ham, among others) and others of low-fidelity such as organic, inorganic, and synthetic simulators ${ }^{2,10,15}$. Although the best simulator for US-guided CVC training should include vessels and also mimic the normal body anatomy with muscles, soft tissues, and bones, there are studies ${ }^{12,20}$ that demonstrate objectively that the technical skills learned on bench models can result in improved performance on animals, human cadavers and in the operating theatre, regardless of the simulator fidelity and thus the choice of a specific simulator should not be based on its fidelity level. Aspects such as availability, seasonal variability, and financial costs should also be considered for this decision (Table 1).

TABLE 1 - Advantages and disadvantages of inanimate bench models for simulation-based ultrasound-guided central venous cannulation skills training $^{2,10,12,15}$.

\begin{tabular}{|c|c|c|c|c|c|c|}
\hline Inanimate bench models & Fidelity & Infection risk & Financial costs * & Availability * & Easy handling & Reutilization ** \\
\hline Parts of postmortem animals & High & Present & +++ & Variable & ++ & Limited \\
\hline Manufactured realistic simulators & High & Absent & +++++ & Variable & +++ & Limited \\
\hline Finger gloves & Low & Absent & + & Extensive & +++ & Limited \\
\hline Homemade ballistic gelatin & Low & Absent & ++ & Extensive & +++ & Limited \\
\hline
\end{tabular}

*varies according to seasonality and geographical region, **limited by risk of infections and/or natural deterioration of material

because although increasing the trainee skill and comfort level, the simulated training does not seem to obviate the need for supervision on the initial CVC by the trainees ${ }^{19}$. It is important that before performing the $\mathrm{CVC}$ on living patients, residents receive adequate training on the US detection of normal vascular anatomy on healthy volunteers. Moreover, as skill maintenance is crucial, periodic testing and refresher training are recommended; at least 10 US-guided CVC should be performed each year in order to maintain proficiency ${ }^{6}$.

\section{Bench models}

As traditional training on fresh human cadavers and living animals involves high costs, limited access, increasing in the risk of infections, specialized installations and also contravenes ethical legal aspects and the use of virtual reality simulators involves high costs and restricted access, several institutions worldwide have been developing alternative inanimate bench models with different applicability ${ }^{12,16,17}$, including the US-guided CVC practice $^{2,10,15}$. These inanimate bench models vary in relation
As the development of an alternative bench model allowing US-guided CVC training in a reliable, reproducible, and inexpensive fashion would be extremely valuable for disseminating and facilitating the US-guided CVC teaching and learning, we adapted a homemade low-fidelity gelatin bench model with inspiration on the ballistic gelatin concept ${ }^{21}$. Ballistic gelatin has physical characteristics that accurately simulate the strength and viscosity of human tissues, making it useful in wound ballistic experiments as a tissue surrogate ${ }^{21}$. Besides being well established as soft-tissue substitute in ballistic tissue injury research ${ }^{21}$, ballistic gelatin has served as an excellent surrogate tissue providing an easily manufactured, reproducible, and inexpensive model for a number of purposes ${ }^{22}$.

In our residency training program, soft-tissues were simulated by means of rectangular $10 \%$ ballistic gelatin blocks prepared in accordance with a standard ballistic gelatin preparation guideline ${ }^{21}$. The ballistic gelatin is composed by double-distilled water, unflavored gelatin (added to 10\%), and a trace amount of pure cinnamon oil $(50 \mu \mathrm{L} / \mathrm{L}$ ) (or $p$-methyl and $p$-propyl benzoic acids) to reduce microbiological 
contamination. The lack of opacity and echogenicity of medium can be improved with the addition of small amount of Metamucil $^{\circledR}$ (dietary fiber supplement) and the firmness of the bench model increases as the concentration of gelatin increases. Subsequently, the prepared $10 \%$ gelatin was poured into the rectangular plastic mold with care taken to minimize air bubble formation. The mould was covered with polyethylene wrap to prevent evaporation and to protect them from airborne microbes. The vessels were simulated by means of rubber tubing (common rubber tourniquet) with two sizes (5 and 10 $\mathrm{mm}$ in diameter) and filled with synthetic blood, red ink or saline; the largest corresponding to the vein and the smallest to the artery. The bench model should be constructed in layers to allow the addition of "vessels": the tubes were suspended in the gelatin solution approximately 1.5 (venous tube) or 2.5 (arterial tube) $\mathrm{cm}$ deep to simulate the internal jugular vein and the carotid artery, respectively. As trainees acquire initial skills, the tubes can be placed and distributed with some overlap to mimic abnormal anatomy. The mould was allowed to solidify in room temperature for $24 \mathrm{~h}$. This lets the gelatin cool off slowly allowing time for any air bubbles to float to the surface before the gelatin solidifies. After that the mould was transferred to a refrigerator at $4^{\circ} \mathrm{C}$ for at least $24 \mathrm{~h}$ for final solidification. The finished bench model was wrapped in plastic wrap (cling film) and stored in a refrigerator until use for many weeks (addition of chlorhexidine solution to the surface of the bench model further prolongs its useful life).

To ensure that the model was both inexpensive and easily attainable, the gelatin and cinnamon oil used were generic brands purchased from commercial outlets, such as craft shops and supermarkets. Any cylindrical material (plastic, elastic, or rubber such as silicone tuning, rubber tubing, cooked penne pasta superglued together, pneumatic pump system, among other) can be used to simulate the vessel. The choice between these cylindrical materials must be cost-based. During the training process we found that the ballistic gelatin bench model could be rapidly and reproducibly generated if the method previously described ${ }^{21}$ was closely followed. All residents involved in the training were able to fabricate their own bench models and bring them to the teaching and learning sessions (Figure 1). Multiple residents can train numerous times on one ballistic gelatin bench model, similar to other low-fidelity simulator ${ }^{10}$. Thus, this homemade bench model is low-cost and reproducible to suit any US-guided CVC training program as previously showed in another environment ${ }^{22}$.

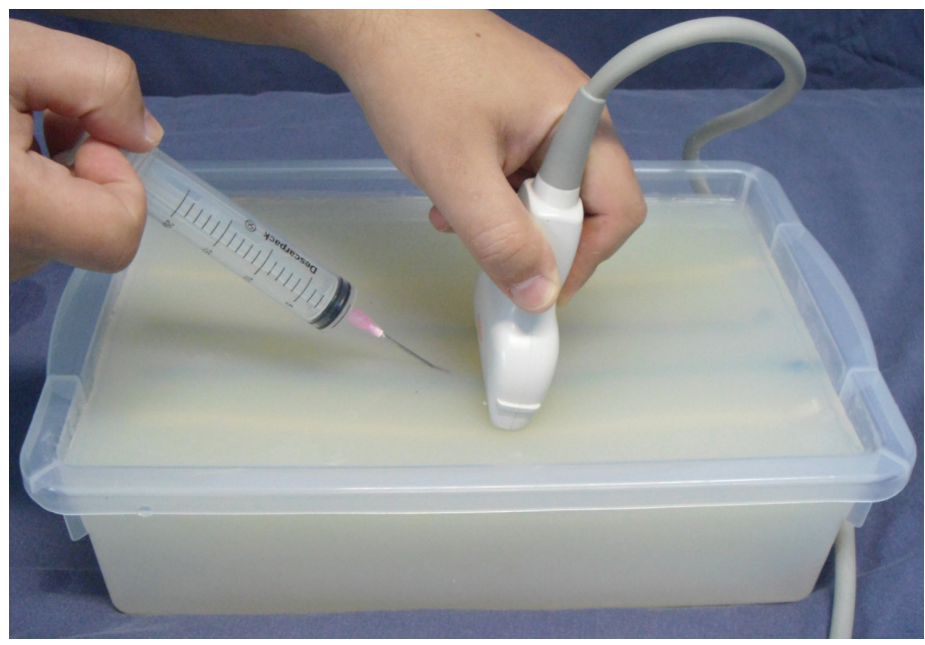

FIGURE 1 - Ultrasound-guided vascular access training on homemade low-fidelity ballistic gelatin bench model with the aid of a linear transducer and a 40x12 needle attached to a $20 \mathrm{ml}$ syringe.

\section{Ultrasound-guided central venous cannulation techniques}

The CVC techniques can be defined as follows: 1) Landmark only: identification of the target vein determined only by anatomy without the use of US; 2) Static US (or US skin mark technique): identification and marking of the target vein by nonsterile US guidance followed by sterile insertion without the use of US guidance during cannulation (or quick-look US of the vessel with a skin mark to guide the needle skin entry point in the direction of the vessel without US guidance); 3) Dynamic US: cannulation of the target vein with direct visualization of the needle entering the vein by a sterile US probe (either in the short or long axis or both); and 4) Landmark failure before US guidance: a failed initial attempt by the landmark technique followed by dynamic US guidance ${ }^{8}$. Here, we will only describe the core concepts on the US-guided CVC technique (static and dynamic approaches).

In US-guided CVC technique, an US probe is used to localize the target vein and to measure its depth beneath the skin ${ }^{1}$. Under US visualization, the introducer needle should be guided through the skin and into the vesse ${ }^{1}$. In this technique, the vein and artery appear black on the US image. The needle appears echogenic and can be followed into the image of the vein on US. Thus, a major advantage of US-guided CVC approach is that the trainee receives visual feedback during simulated vascular cannulation ${ }^{4}$. The needle trajectory can be visualized and adjusted in real time to ensure a direct approach to the vessel of interest with no risk of puncture of nearby vital structures ${ }^{4}$. 


\section{Aseptic technique}

To the placement of central venous catheters, use aseptic techniques (e.g., trainee aseptic preparation and patient skin preparation) and maximal barrier precautions (e.g., sterile gowns, sterile gloves, caps, masks covering both mouth and nose, and full-body patient drapes) should be the focus of the training process because it reduces significantly infections associated with $\mathrm{CVC}^{5}$. For US-guided $\mathrm{CVC}$, preparatory steps are the same as with any vascular access procedure, with the added step of US equipment preparation. So, to US-guided CVC a sterile barrier is typically also needed. Such barriers usually are designed to cover both the transducer and its cable and allow sterile performance by a single operator. Although there are many relatively inexpensive varieties of sterile transducer covers, occasionally, one may not be available. In this situation, other alternatives can be employed. The easiest method is to use a sterile glove or any sterile plastic material (e.g., adopted in laparoscopic surgery). A sterile conductive medium (e.g., xylocaine gel or US gel) is placed inside the glove/plastic wherever the largest uninterrupted flat surface is located. An assistant can then place the transducer inside the glove/plastic. The trainee then folds back the fingers of the glove and holds the transducer so that the flat surface of the glove forms the scanning surface for the transducer; with the plastic material, this is simpler. It is important to eliminate any air bubbles that may be interposed between the glove/plastic and the transducer's scanning surface because they would compromise image quality severely ${ }^{2}$. Additionally, the sterile sheaths are also necessary ${ }^{6}$.

\section{Understanding ultrasound technology}

All trainees should have dominion over the core concepts inherent to the use of US technology:

Ultrasound machine: Several US machines are available, each with their own advantages and disadvantages. The level of sophistication of US equipment varies widely, but even the most basic models are usually sufficient for US-guided CVC. Peripherals such as larger monitors, docking stations, and needle guides may be nice to have but are not essential ${ }^{6}$. Trainees should gain familiarity with the specific functions of the US machine in use at their own institution.

Applied physics: US is defined as any sound with a frequency $>20 \mathrm{kHz}$ and for medical imaging this is typically within the range $3-15 \mathrm{MHz}$. The US pulse is generated by applying an electric voltage to pieces of electric crystals within the probe, which is directed into tissues. The echo is produced by reflected sound waves returning to the probe deforming the crystals, to produce an electrical pulse (the echo). By convention, no reflection is displayed as black (anechoic) and a strong reflection as white (hyperechoic). Multiple echoes are produced by multiple repeated pulses generated by an array of crystals in the ultrasound probe, allowing a real-time image to be generated $^{6}$.

Ultrasound transducer: Proper probe selection is critical for achieving high image quality. The relationship between US transducer frequency and the depth of tissue penetration is an inverse relationship. Low-frequency US (1-3 $\mathrm{MHz}$ ) penetrates more deeply than high-frequency US (7-10 $\mathrm{MHz}$ ). The relationship between frequency and image detail (or resolution) is proportional. Thus, high-frequency US provides a very detailed image of superficial structures, to a depth of approximately $5 \mathrm{~cm}$, but cannot penetrate into deeper tissues. For vascular access, one should use a higher-frequency linear array transducer because this provides excellent resolution and sufficient penetration ${ }^{6}$. A linear (flat) shape is ideal for imaging veins because it allows the trainee to apply even compression when distinguishing arteries from veins. A curved transducer with a lower frequency range may be useful in obese patients for increased penetration of tissues. A "hockey-stick" transducer (a type of linear transducer) is appropriate for tight locations such as the neck in a small child.

Modes ultrasound: Two types of US guidance are available: B- and Doppler-modes. B-mode (brightness mode) US uses an US probe with many active elements aligned in a specific orientation, or "array," to create a standard 2D grayscale image of the tissue. Doppler-mode transforms the sound waves reflected from a moving object (e.g., blood) into an amplified audio signal. Although there is clinical interest in three-dimensional (3D) US for vascular access, 2D imaging is currently the standard technology for US-guided CVC. Doppler imaging is regarded as an advanced US skill without improving success ${ }^{4,6}$.

Static versus dynamic ultrasound guidance: US guidance can be categorized into two ways: static (or indirect) and dynamic (or direct) approaches. Static guidance refers to identifying the target vessel, assessing patency, and marking an appropriate insertion site with US, then cannulating "blindly". This approach has the following characteristics: ultrasonic localization and marking of landmarks only; cannulation is not image guided; time delay between marking and cannulation; less 
difficult to maintain sterility; and less technically demanding. Dynamic guidance refers to performing the procedure in "real time" with US imaging viewing the needle puncturing the vessel wall. This approach has the following characteristics: ultrasonic localization and image-guided cannulation; more precise and "real time"; more difficult to maintain sterility; more technically demanding; and it requires significant handeye coordination. Although there are such advantages and disadvantages, different guidelines strongly recommend the real-time, dynamic method of US guidance for CVC because static guidance appears to be inferior to dynamic guidance but still better than the landmark-based technique alone $e^{2,5}$.

Sonographic ultrasound probe orientation: Each US probe has a marker (denoted by a light or a notch on the side of the probe) placed on one side which correlates to a mark on the image screen. This marker allows the trainee to orient the probe and thus allow for uniformity of imaging. By convention, structures beneath the left aspect of the US probe marker are always displayed on the left side of the imaging screen. With this in mind, there are two standardized scanning planes: longitudinal (or sagittal) and transverse. In the longitudinal plane, the probe is oriented with the marker towards the patients head, providing a view of the vein along its long axis. In the transverse plane, which provides an image similar in appearance to a computed tomography scan, the probe is placed with the marker facing towards to the patient's right. In the transverse plane, the vessels appear round and not tubular, as if cut in cross-section ${ }^{2,6}$. In simulated training, it is important to determine which side of the model is the "head" and which the "trunk". So the trainee learns aspects related to sonographic US probe orientation.

Short-axis versus long-axis approach: Using basic B-mode imaging, two US-guided CVC techniques are used: the short-axis (out-of-plane) approach and the longaxis (in-plane) approach, which are defined according to the relationship between the needle direction and the direction of the US scanning plane. The short-axis technique aligns the two directions in the perpendicular (or transverse) fashion (i.e., needle is "out-of-plane"), while the long-axis technique aligns them in the longitudinal (or parallel) direction (i.e., needle is "in-plane"). In short-axis approach, the vessel should appear as an anechoic circle on the screen with the needle visualized as a hyperechoic point in cross section (Figure 2, Left), while in long-axis approach, the image should show the course of the vessel across the screen and the shaft and point of the needle as it is advanced (Figure 2, Right) ${ }^{2,4}$.
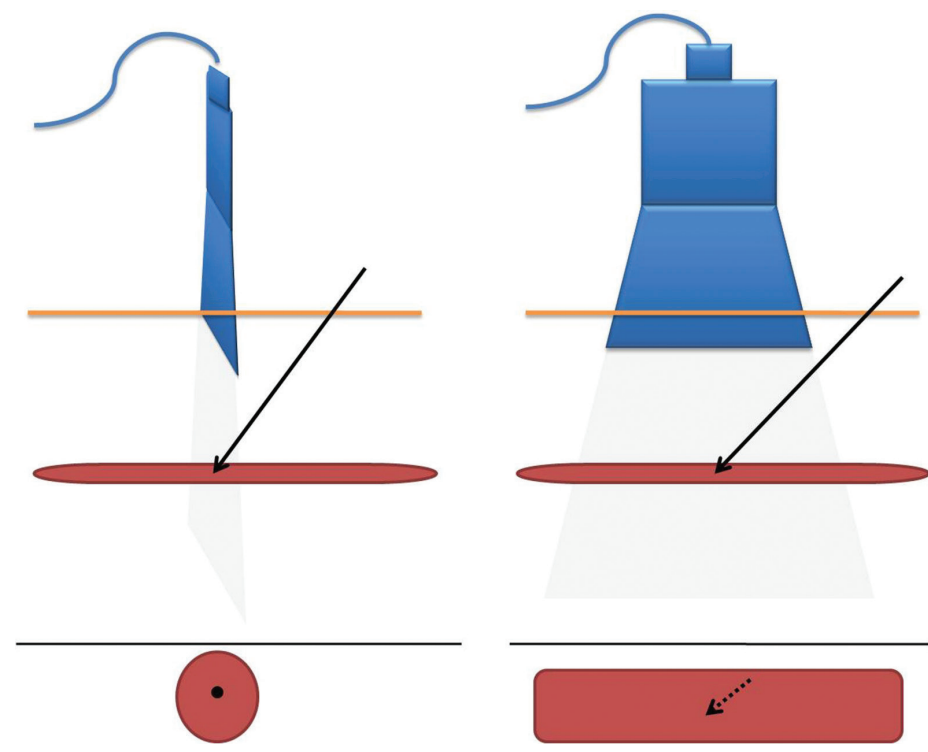

FIGURE 2 - Illustration depicts the transducer orientation with basic axial planes. Note the positioning of the probe to obtain a (Left) transverse view (or short-axis) or a (Right) longitudinal view (or long-axis) of the vessel punctured by the needle (arrows). (Below) The needle is kept in cross-sectional view or entirely in the field of view in the short-axis and long-axis approaches, respectively.

The advantages of the short-axis over the long-axis approach are that it is easier to perform in anatomic areas where space is limited (e.g., neck), and inexperienced trainees can acquire proficiency more quickly. On the other hand, performing the procedure along the vessel's long-axis allows much better visualization of the advancing needle tip (a trainee could constantly monitor the tip - or entire length - of the needle under US image) and, therefore, may avoid inadvertent punctures of the posterior vessel wall ${ }^{2,4}$. Thus, as the utilization of the long-axis (in-plane) technique leads to higher precision and fewer complications (posterior vessel wall perforations and punctures of neighboring organs), it should be used when possible, although it is more challenging (there is a technical difficulty in maintaining the linear relationship between the needle and the US scanning plane) $)^{4}$. Alternatively, as the short-axis approach is adequate and sufficient to follow needle tip location, it may be helpful to start a procedure with a short-axis view to assure that the needle is centered over the middle of the vessel and then rotate the probe to a long-axis view as the needle is advanced. Thus, adequate training for US-guided CVC should include knowledge and practice of both techniques ${ }^{4}$.

One-person versus two-person dynamic approach: USguided CVC may be performed as a one-person or a two-person dynamic approach. Two-person dynamic approach (one person to perform the US while another person performs the procedure) provides the potential advantage of allowing the person performing 
the procedure to use two hands for the procedure itself and does not require the dual hand-eye coordination of directing the US transducer as well as performing the procedure. However, the two-person approach has the disadvantage of requiring additional personnel, and it may be more difficult for the trainee doing the procedure to optimize the image positioning relative to the needle. In a oneperson dynamic approach the person performing the procedure holds the needle with one hand while directing the US probe in the other hand. While a one-person dynamic approach requires more experience, it is preferred by most advanced practitioners as it allows for real-time hand-eye coordination ${ }^{6}$. During the training phases, both techniques should be taught and practiced. In clinical practice, the use of each of them must be based on the availability of physicians as well as the individual skills.

\section{Performing the procedure}

After mastering the principles described above in an isolated manner, trainees must perform the entire procedure. Here, some of the technical principles of US-guided CVC technique are described. Prior to needle insertion, the axis of the vessel should be noted and the expected direction of the needle path should be in-line with this axis:

In the short-axis approach (Figure 3, Left), the vessel is identified in the transverse plane (Figure 4, Left) and centered under the transducer. The midpoint of the transducer then becomes a reference point for insertion of the needle. The needle is inserted at a $45^{\circ}$ angle to the transducer (Figure 5). As the needle is advanced, the tip is visualized as it approaches the anterior wall of the vessel. After contacting the anterior wall of the vessel, further insertion of the needle will cause posterior displacement of the vessel wall. A flash of blood in the syringe signifies that the needle has entered the vessel and then standard catheter placement continues ${ }^{2,14}$.
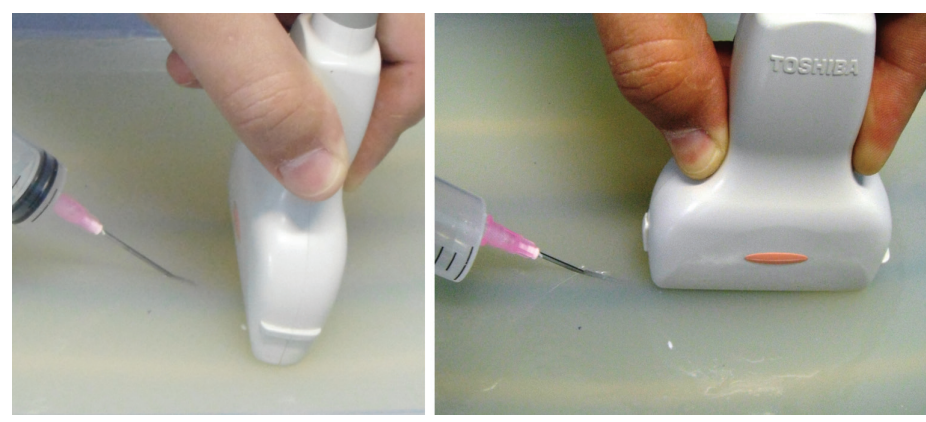

FIGURE 3 - (Left) Short-axis approach (perpendicular view of the simulated vessel) and (Right) long-axis approach (parallel view of the simulated vessel) on homemade low-fidelity ballistic gelatin bench model.
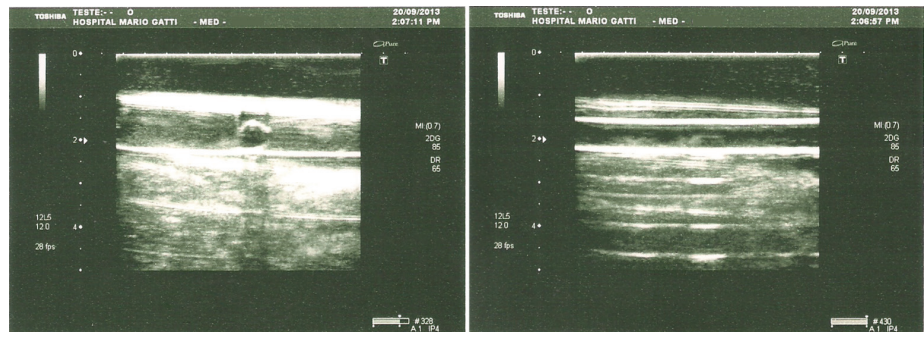

FIGURE 4 - Simulated vessels on ultrasound image. (Left) Round and (Right) tubular shapes on the screen related to short-axis and long-axis approaches, respectively.

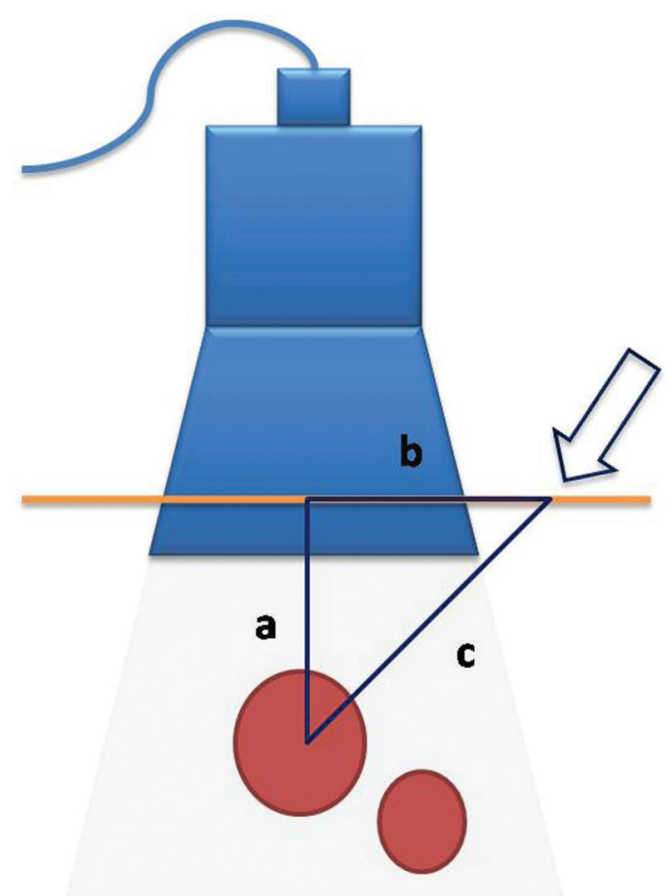

FIGURE 5 - Illustration depicts the short-axis approach. In this technique, the probe should be placed on top of the skin and the simulated vessel in the middle of the display. To assess trajectory and distance of the needle to puncture the simulated vessel, the principles of the Pythagorean Theorem $\left(a^{2}+b^{2}=c^{2}\right)$ should be adopted. For example, if the simulated vessel is 1 $\mathrm{cm}$ deep (a), it is need to puncture (arrow) the skin $1 \mathrm{~cm}$ away from the transducer (b) at a $45^{\circ}$ angle. The simulated vessel will be then punctured at $1.4 \mathrm{~cm}(\mathbf{c})$. In any isosceles right triangle, the hypotenuse (c) is 1.4 times the length of the equilateral sides (a and $\mathbf{b}$ ).

In the long-axis approach (Figure 3, Right) it identifies the vessel in its long axis (Figure 4, Right) and involves lining up the transducer over the greatest anterior-posterior diameter of the vessel. The needle is then inserted through the skin just off one end of the transducer in a plane that is in line with the long axis of the transducer and at an approximate $45^{\circ}-60^{\circ}$ angle to the skin surface. As the needle is advanced, its progress through the subcutaneous tissue is monitored in real-time on the US screen. After the needle has punctured the anterior wall of the vessel and a flash of blood is apparent in the syringe, standard catheter placement should continue ${ }^{2}$. 
The short-axis view allows the needle approach to be over the center of the vein. However, care must be taken to "fan" the plane of the image along the needle as it advances in order to track the tip and avoid underestimating the depth of the tip. While the long-axis view has the advantage of allowing visualization of the entire needle shaft and tip, it may be more difficult to keep the plane of the US in line with the vessel as the plane of the image may "slip off" to the side of the plane of the needle and/or the center of the vessel ${ }^{3}$.

Once the needle has entered the vessel in both techniques, advancement and correct positioning of the guidewire may be aided by US visualization. Placement of the guidewire, dilator, and line may then proceed with direct visualization of the guidewire/ dilator within the vein can ensure proper placement ${ }^{3}$.

\section{Common pitfalls in ultrasound-guided central ve- nous cannulation technique}

The most common pitfalls encountered in US-guided CVC include not understanding the relationship between transducer frequency and both depth of penetration and image resolution, poor technique in terms of always keeping the needle tip in view during image-guided cannulation, not paying attention to equipment setup to maximize comfort and ergonomics during the procedure, and not scanning the entire vessel to exclude the presence of thrombus. Another common pitfall is applying too much pressure with the transducer, thus collapsing the target vessel and rendering it invisible. All these pitfalls can be avoided by proper training and subsequent practice ${ }^{1-4}$.

\section{Feedback from instructors}

As feedback from instructors is associated with a better and faster learning and also with greater knowledge retention over time, all trainees should receive feedback during and at the end of each training stage supervised by instructors, or in specific times scheduled after the self-directed training ${ }^{17}$. Instructors must analyze specific movements, paying attention to inadequacies (e.g., technical errors such as breaks in sterile technique, improper positioning of the materials, removal of the hand from the guidewire during any portion of the procedure, inability to thread the wire, inability to flush all ports, inability to handle the US technology, among others), and following this, they should provide a constructive feedback (to point and to correct any technical errors) to trainees ${ }^{8}$. Thus, trainees improve skills based on their mistakes and can be practiced repeatedly, having, as a result, a gain of skills over time ${ }^{13}$.

Trainees should be distributed around rectangular tables, providing mobility to the instructor to clarify any doubts individually and also in subgroups ${ }^{16,17}$. The adoption of a 1:4 (or 1:5) instructor: trainee ratio is recommended to maximize the hands-on training ${ }^{18}$. Additionally, as the number of faculty surgeons for simulation-based training is scarce, all feedback process can be given by attending physicians, surgical fellows, or residents without compromising the learning ${ }^{8,9}$. In our residency training program, senior surgical residents (second- or -third year) play an important role in the teaching and supervision of US-guided CVC for junior general surgical residents (first-year), similar to what was demonstrated in a recent study ${ }^{9}$ conducted at two Canadian residency programs.

\section{Assessment and certification}

In previous studies ${ }^{16,17,20}$, the importance of an objective evaluation during and at the end of the whole simulation-based practice process of each proposed technical skill in order to measure the level of acquisition of the taught skills has been emphasized. Several objective assessment tools have been described worldwide ${ }^{54}$. As Global Rating Scale (GRS) has the advantage of being used to assess generic aspects of technical performance and it has a broad applicability, without the need of developing specific lists for each procedure, this scale has been adopted as a measurement and certification tool in several surgical reports ${ }^{16,17,20,23}$. With the adapted nine-item GRS (Table 2), it is possible to evaluate the performances of trainees in ninth main areas, through a six-point Likert scale for five areas and a five-point scale for four areas, being one the minimum score and six (or five) the maximum one, so that the maximum score achieved is fifty ${ }^{23}$. Instructors can apply this scale at the end of each training session, and in subsequent sessions, they follow the gain of skills and specific points (among the ninth evaluated ones) that deserve attention. 
TABLE 2 - Global Rating Scale ${ }^{23}$ used to assess qualitatively and objectively all two-dimensional ultrasound-guided central venous cannulations during the teaching and learning process.

\begin{tabular}{|c|c|c|c|c|c|c|}
\hline \multicolumn{7}{|c|}{ Please rate the candidate's performance on the following scale: } \\
\hline $\begin{array}{l}\text { Appropriate preparation } \\
\text { of instruments preprocedure }\end{array}$ & 1 & 2 & 3 & 4 & 5 & NA \\
\hline & $\begin{array}{l}\text { Not competent to perform } \\
\text { independently }\end{array}$ & $\begin{array}{l}\text { Borderline competence to } \\
\text { perform independently }\end{array}$ & $\begin{array}{l}\text { Competent to perform } \\
\text { independently }\end{array}$ & & $\begin{array}{l}\text { Above average competence } \\
\text { to perform independently }\end{array}$ & \\
\hline \multirow[t]{2}{*}{ Appropriate analgesia } & 1 & 2 & 3 & 4 & 5 & NA \\
\hline & $\begin{array}{l}\text { Not competent to perform } \\
\text { independently }\end{array}$ & $\begin{array}{l}\text { Borderline competence to } \\
\text { perform independently }\end{array}$ & $\begin{array}{l}\text { Competent to perform } \\
\text { independently }\end{array}$ & & $\begin{array}{l}\text { Above average competence } \\
\text { to perform independently }\end{array}$ & \\
\hline \multirow[t]{2}{*}{ Time and Motion } & 1 & 2 & 3 & 4 & 5 & NA \\
\hline & Many unnecessary moves & & $\begin{array}{l}\text { Efficient but some } \\
\text { unnecessary moves }\end{array}$ & & $\begin{array}{l}\text { Economy of movement \& } \\
\text { maximum efficiency }\end{array}$ & \\
\hline \multirow[t]{2}{*}{ Instrument handling } & 1 & 2 & 3 & 4 & 5 & NA \\
\hline & $\begin{array}{l}\text { Repeatedly makes tentative } \\
\text { and awkward moves }\end{array}$ & & $\begin{array}{l}\text { Competent, occasionally } \\
\text { appeared stiff or awkward }\end{array}$ & & $\begin{array}{c}\text { Fluid movements, } \\
\text { no awkwardness }\end{array}$ & \\
\hline \multirow[t]{2}{*}{$\begin{array}{l}\text { Flow of procedure } \\
\text { and forward planning }\end{array}$} & 1 & 2 & 3 & 4 & 5 & NA \\
\hline & $\begin{array}{l}\text { Frequently topped or needed } \\
\text { to discuss next move }\end{array}$ & & $\begin{array}{l}\text { Demonstrated ability for } \\
\text { forward planning }\end{array}$ & & $\begin{array}{l}\text { Obviously planned course with } \\
\text { effortless flow }\end{array}$ & \\
\hline \multirow[t]{2}{*}{ Knowledge of instruments } & 1 & 2 & 3 & 4 & 5 & NA \\
\hline & $\begin{array}{c}\text { Frequently used an } \\
\text { inappropriate instrument }\end{array}$ & & $\begin{array}{l}\text { Used appropriate } \\
\text { instrument for the task }\end{array}$ & & $\begin{array}{l}\text { Obviously familiar with } \\
\text { required instruments }\end{array}$ & \\
\hline \multirow[t]{2}{*}{ Aseptic technique } & 1 & 2 & 3 & 4 & 5 & NA \\
\hline & $\begin{array}{l}\text { Not competent } \\
\text { to perform } \\
\text { independently }\end{array}$ & $\begin{array}{l}\text { Borderline competence to } \\
\text { perform independently }\end{array}$ & $\begin{array}{l}\text { Competent to perform } \\
\text { independently }\end{array}$ & & $\begin{array}{l}\text { Above average competence to } \\
\text { perform independently }\end{array}$ & \\
\hline \multirow[t]{2}{*}{$\begin{array}{l}\text { Seeks help where } \\
\text { appropriate }\end{array}$} & 1 & 2 & 3 & 4 & 5 & NA \\
\hline & $\begin{array}{l}\text { Not competent } \\
\text { to perform } \\
\text { independently }\end{array}$ & $\begin{array}{l}\text { Borderline competence to } \\
\text { perform independently }\end{array}$ & $\begin{array}{l}\text { Competent to } \\
\text { perform } \\
\text { independently }\end{array}$ & & $\begin{array}{l}\text { Above average competence } \\
\text { to perform independently }\end{array}$ & \\
\hline \multirow[t]{2}{*}{$\begin{array}{l}\text { Overall ability } \\
\text { to perform procedure }\end{array}$} & 1 & 2 & 3 & 4 & 6 & NA \\
\hline & $\begin{array}{l}\text { Not competent } \\
\text { to perform } \\
\text { independently }\end{array}$ & $\begin{array}{l}\text { Borderline competence to } \\
\text { perform independently }\end{array}$ & $\begin{array}{l}\text { Competent to perform } \\
\text { independently }\end{array}$ & & $\begin{array}{l}\text { Above average competence } \\
\text { to perform independently }\end{array}$ & \\
\hline
\end{tabular}

NA, not applicable

Since all trainees must reach mastery standards in a simulated environment, before performing procedures on living patients, GRS can also be used as a certification tool $1^{16,17,20,23}$. To this end the scores of the ninth summary item ("overall ability to perform procedure") should be dichotomized such that a score of 3 or more was considered "competent to perform the procedure", while a score of two or less was considered "not competent to perform the procedure" ${ }^{23}$. In fact, according The Royal College of Radiology "different trainees

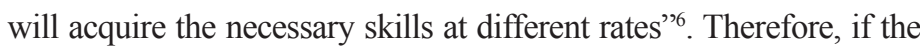
trainee meets the predefined criteria based on objective assessment, he/she can progress to the next stage of training (considered as a more complex one). However, if the trainee is not able to proceed, the training process should be repeated and focused on specific deficits, and, then, a new objective assessment should be carried out ${ }^{16,17,20}$.

\section{Discussion}

In most residency programs, residents-in-training learn CVC by the apprenticeship model "see one, do one, teach one" ${ }^{\prime 9,23}$. However, in the last two decades, the efficiency, effectiveness and ethics of this training model have been increasingly questioned $^{19}$. Additionally, there is an emerging consensus that it is no longer acceptable to use living patients to gain this early experience ${ }^{12,16,17,20}$. So, this apprenticeship method has been replaced by a simulation-based teaching model ("see one, simulate many, do one competently, and teach everyone") ${ }^{12,16,17,19,20}$. As while providing the risk-free training environment, simulation-enhanced training may both accelerate the learning curve and facilitate introduction of living patients at a safer level of performance ${ }^{13}$, 
over the last decade, the simulation-based technical skills' training has been increasingly used in various medical specialties within medical education ${ }^{12,16,17,20}$. It is not different in field in a commonly performed procedure such as $\mathrm{CVC}^{10,13}$.

In this context, as the domain of US-guided CVC should be part of the armamentarium of all residents-in-training and it is recommended that the acquisition of this skill occurs outside the real environment (simulation-based training) before any procedures on living patients ${ }^{9}$, the main focus of this study was to propose a USguided CVC skills' teaching program during residency through the practice on low-fidelity bench models. In order to increase the armamentarium of technical skills of residents-in-training, this proposal can be incorporated and adapted in order to complement the teaching and learning methodologies already established in different residency programs. Moreover, the principles covered in this program can be incorporated in the training of medical students during the undergraduate medical formation.

Although simulation-based education allows trainees an opportunity for deliberate practice without jeopardizing patient safety, some barriers such as costs, availability of space, material resources, and faculty surgeon availability, and time to the implementation of a simulation-based training program have been described as factors affecting the implementation of this teaching and learning apprenticeship model ${ }^{4}$. New alternatives must then be developed.

The problem of cost could be addressed by sharing an US machine with another hospital department, or may be minimized by the introduction of US machines into emergency departments for other indications such as trauma scanning ${ }^{3}$. In our residency training program we have used an US machine that was replaced by the radiology department for a newer model. So, residents manipulate the US machine according to need, as many times as necessary without interfering with the functioning of the remainder of the institution.

Another way to reduce costs partly is adopting low-cost bench models ${ }^{12,10,16,17,20}$, as used herein. A variety of bench models has been described for similar purposes; there are those with high fidelity and other with low-fidelity $2,10,15$. Despite the intuitive belief that "the more realistic, the better" within the scope of simulation-based education, several authors ${ }^{12,20}$ have demonstrated objectively that the technical skills acquired on bench models are independent of fidelity. Moreover, as it was also demonstrated that the transfer of technical skills to the clinical environment is independent of fidelity of the simulator used as a teaching tool ${ }^{12}$, the choice of a particular model should not be based solely upon their fidelity. Other requirements such as availability, versatility, reproducibility, minimal need for storage, and costs should be considered in this choice ${ }^{10,12,17,20}$.
In this context, high fidelity bench models prepared with parts of postmortem animals have limited use due to the need for structure, space and adequate storage conditions ${ }^{10,12,15,16,17,20}$. On the other hand, as the low-fidelity simulators are relatively cheap, simple, portable, reproducible and versatile, of easy accessibility and handling, they can provide more material for repetitive training in any environment ${ }^{2,10,15}$, without compromising the results ${ }^{12,20}$. Thus, in this US-guided CVC training program, adopted a modified homemade low-fidelity ballistic gelatin bench model as teaching platform because this enable the understanding of tridimensional US-guided CVC and it is also extremely lowcost when compared to other manufactured simulators such as Ultrasound Central Venous Catheter Insertion Simulator Mk 2 (Limbs \& Things ${ }^{\circledR}$ ), Ultrasound Vascular Access Trainer (Limbs \& Things $^{\circledR}$ ), CentraLineMan System $\left(\right.$ Simulab $\left.^{\circledR}\right)$, Central Line Training Package $\left(\right.$ Simulab $\left.^{\circledR}\right)$, among others ${ }^{10}$. Moreover, these manufactured simulators have greater difficulty of access.

A subtle difference between the bench model described here and other similar previously presented simulators ${ }^{2,10}$ is that our modified bench model followed a standard guideline for the production of ballistic gelatin ${ }^{21}$. Thus, besides the ease of manufacture and reproduction, homogeneous quality can be easily reached by anyone (resident-in-training or instructor) who is preparing the simulator based on the ballistic gelatin concept ${ }^{21}$. These gelatin bench models suffer from lack of opacity and echogenicity and it can be reduced with the use of graphite powder, millet flour, or dietary fiber supplement, as in our modified bench model.

Although costs can be reduced with the adoption of low cost bench models and with the use of alternative US machines, the availability of time remains a problem for faculty surgeons ${ }^{17}$. Therefore, the incorporation of senior residents emerges as an alternative that can reduce the number of faculty surgeons who are transferred from patient care for simulation environments without compromising learning ${ }^{9}$. For this, all educational efforts should focus on preparing residents for their role in teaching and supervision of US-guided $\mathrm{CVC}^{9}$. With this measure, faculty surgeons would focus on teaching complex tasks and cognitive aspects of surgical education (decision making in the operating theater). Encouraging self-practice can also help reduce the supervised teaching time ${ }^{16,17,20}$.

Within the simulation-based surgical education, training programs must be able to objectively evaluate the gain of technical skills during formation ${ }^{16,17,20,23}$. Among the various objective methodologies described, the Objective Structured Assessment of Technical Skills (OSATS) which is composed of a Task-Specific Checklists (TSC) and a GRS has been considered the gold standard tool $^{17,20,23}$. In this training program, we adopt only the GRS as an 
instrument of evaluation and certification of the training process, because it was demonstrated that the GRS used alone has better results (reliability and validity) when compared with TSC alone or associated with $\mathrm{GRS}^{24}$. Moreover, according to a recent report ${ }^{23}$, the adapted nine-item GRS should be preferred for the assessment of CVC skills using simulation when compared with checklists.

In the present study, several key attributes (e.g., a curriculum clearly describing the technical approach and cognitive elements required; hands-on simulation training to develop handeye skills; and CVC supervised by instructors giving feedback for improvement) for teaching safe US-guided $\mathrm{CVC}^{1-4}$ were include. However, additional details about the complete procedure on living patients and a full consideration on indication, site selection, and management of complications related to CVC are beyond the scope of this article and were detailed elsewhere ${ }^{1-4}$. Additionally, this study does not meet all the needs of residentsin-training, which should include the acquisition of other bedside procedural skills such as venous cutdown, chest tube thoracostomy, pericardiocentesis, paracentesis, cricothyrotomy, among other.

\section{Conclusion}

The proposal of simulation-based US-guided CVC training is a further complementary alternative to the armamentarium of residency training programs already established in order to better prepare residents before their contact with living patients which remains as the cornerstone of medical education.

\section{References}

1. McGee DC, Gould MK. Preventing complications of central venous catheterization. N Engl J Med. 2003;348(12):1123-33.

2. Abboud PA, Kendall JL. Ultrasound guidance for vascular access. Emerg Med Clin North Am. 2004;22(3):749-73.

3. Atkinson P, Boyle A, Robinson S, Campbell-Hewson G. Should ultrasound guidance be used for central venous catheterisation in the emergency department? Emerg Med J. 2005;22(3):158-64.

4. Lamperti M, Bodenham AR, Pittiruti M, Blaivas M, Augoustides JG, Elbarbary M, Pirotte T, Karakitsos D, Ledonne J, Doniger S, Scoppettuolo G, Feller-Kopman D, Schummer W, Biffi R, Desruennes E, Melniker LA, Verghese ST. International evidencebased recommendations on ultrasound-guided vascular access. Intensive Care Med. 2012;38(7):1105-17.

5. American Society of Anesthesiologists Task Force on Central Venous Access, Rupp SM, Apfelbaum JL, Blitt C, Caplan RA, Connis RT, Domino KB, Fleisher LA, Grant S, Mark JB, Morray JP, Nickinovich DG, Tung A. Practice guidelines for central venous access: a report by the American Society of Anesthesiologists Task Force on Central Venous Access. Anesthesiology. 2012;116(3):539-73.

6. Feller-Kopman D. Ultrasound-guided internal jugular access: a proposed standardized approach and implications for training and practice. Chest. 2007;132(1):302-9.
7. O'Grady NP, Alexander M, Burns LA, Dellinger EP, Garland J, Heard SO, Lipsett PA, Masur H, Mermel LA, Pearson ML, Raad II, Randolph AG, Rupp ME, Saint S; Healthcare Infection Control Practices Advisory Committee. Guidelines for the prevention of intravascular catheter-related infections. Am J Infect Control. 2011;39(4 Suppl 1):S1-34.

8. Dodge KL, Lynch CA, Moore CL, Biroscak BJ, Evans LV. Use of ultrasound guidance improves central venous catheter insertion success rates among junior residents. J Ultrasound Med. 2012;31(10):1519-26.

9. Ma IW, Teteris E, Roberts JM, Bacchus M. Who is teaching and supervising our junior residents' central venous catheterizations? BMC Med Educ. 2011;11:16.

10. Di Domenico S, Santori G, Porcile E, Licausi M, Centanaro M, Valente U. Inexpensive homemade models for ultrasound-guided vein cannulation training. J Clin Anesth. 2007;19(7):491-6.

11. Evans LV, Dodge KL, Shah TD, Kaplan LJ, Siegel MD, Moore CL, Hamann CJ, Lin Z, D'Onofrio G. Simulation training in central venous catheter insertion: improved performance in clinical practice. Acad Med. 2010;85(9):1462-9.

12. Reznick RK, MacRae H. Teaching surgical skills-changes in the wind. N Engl J Med. 2006;355(25):2664-9.

13. Dong Y, Suri HS, Cook DA, Kashani KB, Mullon JJ, Enders FT, Rubin O, Ziv A, Dunn WF. Simulation-based objective assessment discerns clinical proficiency in central line placement: a construct validation. Chest. 2010;137(5):1050-6.

14. Flato UA, Petisco GM, Santos FB. Ultrasound-guided venous cannullation in critical care unit. Rev Bras Ter Intensiva. 2009;21(2):190-6.

15. Miranda RB, Nardino EP, Gomes T, Farias P. New technique for ultrasound-guided vascular access training using an animal tissue model. J Vasc Bras. 2012;11(1):83-7.

16. Bastos EM, Silva RD. Proposal of a synthetic ethylene-vinyl acetate bench model for surgical foundations learning: suture training. Acta Cir Bras. 2011;26(2):149-52.

17. Denadai R, Souto LR. Organic bench model to complement the teaching and learning on basic surgical skills. Acta Cir Bras. 2012;27(1):88-94.

18. American College of Emergency Physicians. Emergency ultrasound guidelines. Ann Emerg Med. 2009;53(4):550-70.

19. Britt RC, Reed SF, Britt LD. Central line simulation: a new training algorithm. Am Surg. 2007;73(7):680-2.

20. Denadai R, Oshiiwa M, Saad-Hossne R. Does bench model fidelity interfere in the acquisition of suture skills by novice medical students? Rev Assoc Med Bras. 2012;58(5):600-6.

21. Jussila J. Preparing ballistic gelatine--review and proposal for a standard method. Forensic Sci Int. 2004;141(2-3):91-8.

22. Mendez-Probst CE, Vanjecek M, Razvi H, Cadieux PA. Ordnance gelatine as an in vitro tissue simulation scaffold for extracorporeal shock wave lithotripsy. Urol Res. 2010;38(6):497-503.

23. Ma IW, Zalunardo N, Pachev G, Beran T, Brown M, Hatala R, McLaughlin K. Comparing the use of global rating scale with checklists for the assessment of central venous catheterization skills using simulation. Adv Health Sci Educ Theory Pract. 2012;17(4):457-70.

24. Regehr G, MacRae H, Reznick RK, Szalay D. Comparing the psychometric properties of checklists and global rating scales for assessing performance on an OSCE-format examination. Acad Med. 1998;73(9):993-7. 
Denadai $R$ et al.

\section{Acknowledgement}

To Dr. Marcelo Eduardo Miguez de Oliveira for his technical support.

\section{Correspondence:}

Rafael Denadai

Departamento de Cirurgia Geral

Hospital Municipal Dr. Mário Gatti

Avenida Prefeito Faria Lima, 340

13036-902 Campinas - SP Brasil

Tel./Fax: (55 14) 3453-5456

denadai.rafael@hotmail.com

Received: Oct 17, 2013

Review: Dec 18, 2013

Accepted: Jan 20, 2014

Conflict of interest: none

Financial source: none

${ }^{1}$ Research performed at Department of Surgery, Hospital Municipal Dr. Mario Gatti (HMMG), Campinas-SP, Brazil. 